\title{
Atualização: Sarcopenia
}

\section{Update: Sarcopenia}

\section{Bruno Prata Martinez ${ }^{1}$ (D) \\ Fernanda Warken Rosa Camelier ${ }^{2}$ (]) Nívia Giullia de Sales e Santos ${ }^{3}$ (1) Laís Vasconcelos Martins da Costa ${ }^{4}$}

\author{
Lindanor Gomes Santana Neta ${ }^{5}$ \\ Joselita Moura Sacramento ${ }^{6}$ (i) \\ Aquiles Assunção Camelier ${ }^{7}$ (1)
}

\footnotetext{
${ }^{1}$ Autor para correspondência. Universidade Federal da Bahia (Salvador). Bahia, Brazil. brunopmartinez@hotmail.com 2-7Universidade do Estado da Bahia (Salvador).Bahia, Brazil.fcamelier@uneb.br, niviagiullia@gmail.com, lais.vasconcelosmcosta@gmail.com,
} Igsneta@uneb.br, jsacramento@uneb.br, aquilescamelier@yahoo.com.br

RESUMO | OBJETIVO: Fazer uma atualização da revisão de literatura sobre sarcopenia publicada em 2014 nesta revista. De acordo com o Consenso do Working Group on Sarcopenia in Older People (EWGSOP2), a sarcopenia foi redefinida como uma doença muscular, caracterizada pela redução da força muscular, associada à diminuição da qualidade/quantidade muscular e/ou desempenho físico, sendo classificada como primária, secundária, aguda e crônica. Além de consequências físicas como aumento da ocorrência de quedas e limitação para atividades cotidianas, pode promover alterações sistêmicas pelo desequilíbrio entre síntese e degradação proteica. A prevalência aumenta com a idade, sendo mais alta a partir de 60 anos. Estudos em seis países encontraram prevalência entre $4,6 \%$ e $22,1 \%$, havendo oscilação de valores conforme definições utilizadas, métodos diagnósticos e os pontos de corte para índice de massa muscular (IMM). Como estratégia para refinar a detecção do risco da sarcopenia, o EWGSOP2 sugere aplicação do questionário SARC-F. Para mensuração da variável massa muscular, os métodos recomendados são Ressonância Magnética, Tomografia Computadorizada, Absorciometria de Raio-X de Dupla Energia, Bioimpedância Elétrica e Antropometria, existindo acurácias e custos variáveis entre eles. Na aferição da força muscular, a principal forma de mensuração é a força de preensão palmar. Já o desempenho físico pode ser quantificado através do teste de velocidade de marcha de quatro metros. As formas de tratamento são treino de exercícios de resistência progressiva e aeróbicos, além de uma nutrição adequada. O estilo de vida sedentário, obesidade e fragilidade são fatores desencadeantes de perda de massa e função muscular no ambiente clínico.
ABSTRACT | OBJECTIVES: To update on a sarcopenia literature review published in 2014 in this journal. According to the Working Group on Sarcopenia in Older People Consensus (EWGSOP2), sarcopenia was redefined as a muscular disease, characterized by muscular strength reduction, associated with a diminished muscular quantity and /or quality and /or low physical performance, being stratified as primary, secondary acute and chronic. Beyond physical consequences as a fall risk and daily activities, sarcopenia can promote a dysbalance between protein synthesis and degradation. Sarcopenia prevalence is higher with increasing age, especially after 60 years. Studies in six countries had found sarcopenia prevalence between $4.6 \%$ and $22.1 \%$, but differences between definitions, diagnostic methods, and cutoff points to evaluate muscle mass and function are found. To improve sarcopenia risk detection, EWGSOP2 suggests the use of the SARC-F questionnaire. Muscle mass measurement recommended methods are Magnet Resonance Imaging, Computed Tomography, Double Energy X-Ray Absorptiometry, Electric Bioimpedance, and Anthropometry with variable accuracy and costs between these methods. To evaluate muscle strength, the handgrip strength test is the main method recommended. In addition, four Meter Gait speed is recommended to evaluate physical performance. Treatment options are progressive exercise, endurance training, and aerobic exercises, together with nutritional interventions. Sedentary lifestyle, obesity, and frailty are the main risks factors associated with muscle mass and function losses in the clinical setting.

KEYWORDS: Sarcopenia; Body composition; Muscular strength.

PALAVRAS-CHAVE: Sarcopenia; Composição corporal; Força muscular. 


\section{Principais pontos abordados na atualização}

- A sarcopenia foi definida como uma doença distinta e recebeu o código CID-10-CM

- O EWGSOP2 revisou, em 2018, os critérios diagnósticos e a definição de sarcopenia, caracterizando-a como uma doença muscular, tendo como principal determinante a redução da força muscular, e sua presença, mesmo isolada, atende à suspeita de sarcopenia

- A sarcopenia está presente quando a redução da força muscular está associada a uma redução na qualidade ou quantidade muscular

- A sarcopenia grave está presente quando a redução da força muscular está associada à redução da qualidade ou quantidade muscular e baixo desempenho físico

- As subcategorias ou classificações associadas ao tempo de duração foram definidos novamente: sarcopenia aguda, associada a lesões ou doenças agudas, e sarcopenia crônica, quando presente por período igual ou superior a 6 meses (que tende a estar relacionada a maior mortalidade).

- Os protocolos incluíram duas ferramentas de triagem: a árvore de decisão Find-Assess-ConfirmSeverity (F-A-C-S) ou Encontrar-Avaliar-ConfirmarGravidade; e o questionário de triagem de risco de sarcopenia, o SARC-F.

\section{Introdução}

O comprometimento da função muscular esquelética, devido ao envelhecimento e por fatores como inatividade física, desnutrição e presença de doenças catabólicas, é um importante problema de saúde pública. ${ }^{1}$ Este relaciona-se a risco aumentado de quedas e fraturas, alterações de mobilidade; $\underline{\underline{2}}$ incapacidade para realizar atividades cotidianas; $;$ associação a doenças cardíacas ${ }^{4}$ e respiratórias ${ }^{5}$ causando diminuição da qualidade de vida, limitações sociais e comprometimento cognitivo $0^{6,7}$, perda de independência ou necessidade de cuidados de longa duração ${ }^{8}$, podendo levar à morte.. Discute-se evidências de que $a$ sarcopenia pode ocorrer em etapas mais precoces da vida, propondo sua detecção através da baixa força muscular. Neste contexto, faz-se necessário aumentar a conscientização sobre a doença, assim como delinear critérios, ferramentas e intervenções para prevenir, retardar, tratar e reverter, quando possível, a sarcopenia.

\section{Histórico e definição}

A sarcopenia foi descrita inicialmente por Rosemberg, como redução da massa muscular global, durante o envelhecimento. Em 1998, Richard Baumgartner desenvolveu uma forma de mensurar a sarcopenia, pautada na determinação da massa muscular relativa ou IMM, pelos métodos de Absorciometria de Raio-X de Dupla Energia (DEXA), onde se divide a massa muscular pela altura ao quadrado, análogo ao cálculo do índice de massa corporal (IMC). Ian Jansen et al., em 2002, propuseram uma classificação baseada na severidade, após avaliação pelo método de bioimpedância elétrica $(\mathrm{BI} A) \cdot 10$

O primeiro Consenso de Sarcopenia foi publicado em 2010 pelo Working Group on Sarcopenia in Older People (EWGSOP) e ampliou sua definição para incluir, além da redução de massa muscular, diminuição de força e piora do desempenho físico, sendo a redução de massa muscular o fator principal de identificação da sarcopenia. ${ }^{11}$

Em 2016, a sarcopenia tornou-se reconhecida como uma doença distinta e recebeu o código CID-10-CM e, em 2018, o EWGSOP2 revisou a definição e o diagnóstico, caracterizando-a como uma doença muscular, sinalizando que a força muscular reduzida é o principal determinante e a presença isolada evidencia suspeita de sarcopenia. ${ }^{1}$ Quando essa força muscular reduzida está associada a diminuição da qualidade ou quantidade de músculo, é possível afirmar a presença de sarcopenia. Nas situações em que há baixa força muscular, baixa qualidade ou quantidade de músculo e baixo desempenho físico é possível afirmar a presença de sarcopenia severa. Em relação ao tempo de duração, também, foram identificadas as subcategorias agudas (associada a lesões ou doenças agudas) e a crônica, quando igual ou superior há 6 meses; a qual tem relação com maior risco de morte. ${ }^{1}$

\section{Causas e consequências}

O principal mecanismo do processo de perda de massa, força e desempenho físico na sarcopenia é o estresse oxidativo, desencadeado por fatores endógenos e exógenos, culminando em redução da síntese 
proteica, aumento da degradação proteica, alteração da integridade neuromuscular e acréscimo do conteúdo de gordura no músculo. $\frac{12}{}$

A sarcopenia primária acomete principalmente os idosos, não havendo outra causa específica evidente e suas alterações revelam a fragilidade, aumento do número de quedas e fraturas, limitação para atividades diárias, podendo influenciar em desfechos negativos na hospitalização e resultar em risco de morte. ${ }^{10}$ Para a secundária, menciona-se a inatividade física que leva ao acúmulo de gordura visceral com ativação de vias inflamatórias, mediadas pela interleucina- 6 , atuantes nas mudanças da composição muscular (redução da produção das miocinas a partir da contração muscular) e diminuição da funcionalidade. $\cdot \underline{13}$

Diversas doenças associadas à falência orgânica avançada, distúrbios inflamatórios e endócrinos podem promover efeitos catabólicos, através de maior degradação proteica. $\frac{14}{}$ Podem estar associadas à sarcopenia, as doenças crônicas (obesidade, insuficiência renal crônica, doença pulmonar obstrutiva crônica (DPOC), diabetes tipo 2, câncer, insuficiência cardíaca congestiva); doenças neurológicas (demência e depressão), fragilidade e desnutrição. ${ }^{13}$

Outro importante fator para a sarcopenia secundária pode estar associado aos aspectos nutricionais, como ingestão inadequada de energia, macro e micronutrientes, desordens gastrointestinais (má absorção), ou uso de medicações que causam anorexia. Ademais, condições semelhantes à sarcopenia como obesidade sarcopênica, fragilidade e desnutrição compreendem um ciclo para agravar suas consequências adversas. ${ }^{1}$

\section{Epidemiologia da sarcopenia}

A prevalência aumenta com a idade e há oscilação de valores conforme definições utilizadas; métodos diagnósticos da massa muscular reduzida, bem como pontos de corte para o IMM. $\frac{15}{}$ Os dados relatam que mundialmente a prevalência é mais alta na população a partir de 60 anos, a despeito de causas multifatoriais presentes no seu aparecimento e progressão. $\frac{16}{}$
Em estudo realizado por Diz et al., que apresentou a prevalência em idosos de seis países: Estados Unidos, Reino Unido, Brasil, Japão, Coreia do Sul e Taiwan, a frequência variou de $4,6 \%$ a $22,1 \%$. Constatou-se alta prevalência em pessoas a partir de 60 anos, sendo as maiores taxas no Japão, correspondendo a $22,1 \%$ no sexo feminino e $21,8 \%$ para o masculino. O Brasil foi o segundo país com proporção elevada, no qual mulheres aparecem com $16,1 \%$, enquanto homens com $14,4 \%$.17

Em investigações nacionais, estudo realizado com idosos de São Paulo encontrou uma prevalência de $4,8 \%$. A razão de risco relativo foi 3,32 para idosos com 70-79 anos e 9,79 para aqueles com 80 anos ou mais. ${ }^{18} \mathrm{Um}$ ensaio desenvolvido em Florianópolis verificou a prevalência de sarcopenia e a associação com mudanças nos fatores socioeconômicos, comportamentais e da saúde, constatando um percentual de $17 \%$ nas mulheres e $28,8 \%$ nos homens. ${ }^{19}$ Já no Rio de Janeiro, a taxa de prevalência na pré-sarcopenia foi de $60 \%$ e na sarcopenia $77,5 \%$ no sexo feminino; enquanto que para o masculino a pré-sacopenia $40 \%$ e a sarcopenia $22,5 \%$. 20

Estudo realizado por Shimokata et al. sobre a epidemiologia da sarcopenia com as doenças crônicas não transmissíveis demonstrou alta prevalência (39,5\%) com diabetes tipo 2. Já na síndrome metabólica, houve uma associação positiva (odds ratio 5,5) à sarcopenia em homens de 65-74 anos, sendo modificada por sexo e idade, contudo a obesidade abdominal foi o principal contribuinte. Em pessoas com DPOC, a prevalência foi de 14,5\%; naquelas infectadas por HIV, 5-24,2\%; com doença renal crônica, 5,9-14\% durante a pré-diálise e $12,7-33,7 \%$ na diálise. ${ }^{15}$

Com relação aos pacientes com neoplasias, foi reportado que o percentual de pré-sarcopenia foi $26-65 \%$ para câncer gástrico/esofágico; 19-39\% no câncer colorretal, 11-66\% em carcinoma hepatocelular; 2163\% câncer pancreático; 29-68\% para câncer renal; 60-68\% câncer de bexiga; cerca de 70\% para câncer de pulmão de células não pequenas; e aproximadamente $55 \%$ no linfoma de célula beta. $\frac{15}{}$

Em uma meta-análise de estudos de coorte prospectivos, evidenciou-se que indivíduos com obesidade sarcopênica tinham $24 \%$ de risco de mortalidade por todas as causas, principalmente os homens. ${ }^{21}$ 


\section{Instrumentos diagnósticos}

São muitas as opções de testes e ferramentas usadas na prática clínica e na pesquisa. A escolha do instrumento depende da mobilidade do paciente, espaço de aplicação, recursos técnicos, e se há uma finalidade de monitoramento da progressão e/ou tratamento do paciente. As variáveis que estabelecem o diagnóstico são força muscular, desempenho físico e quantidade ou qualidade do músculo. Essa era a variável de relevância; entretanto, a força muscular passou a ter destaque, devido à sarcopenia ser considerada doença muscular. ${ }^{1}$

Para a prática clínica deve-se examinar as queixas e percepções aparentes, atentando-se para as quedas, fraqueza, marcha lenta, dificuldade em levantar-se da cadeira e redução de peso e massa muscular. ${ }^{-}$Neste contexto, o questionário SARC-F é recomendado para triagem do risco, por ser acessível, possuir uma sensibilidade de baixa a moderada, além de especificidade muito alta para prever a baixa força muscular. É autorrelatado e versa sobre as limitações da força, habilidade de andar, levantar-se da cadeira, subir escada e histórico de quedas..$^{1-3}$ O teste Ishii pode ser utilizado, por fazer cálculo baseado na idade, força de preensão e circunferência da panturrilha. ${ }^{1}$

Como parâmetros de mensuração da sarcopenia, encontram-se:

\section{a) Força muscular}

A força de preensão palmar é a mais empregada, obtida pela dinamometria manual.22 $\mathrm{Na}$ identificação de fraqueza muscular os valores de referência para muIheres são inferiores a $20 \mathrm{~kg}$ e nos homens inferiores a $30 \mathrm{~kg} .{ }^{1}$ Todavia, existem circunstâncias em que sua aplicação é inviável, como artrite avançada e acidente vascular cerebral, sendo aplicados métodos de torque isométrico para medição da força nos membros inferiores. 23
O teste de levantar-se da cadeira como substituto para determinar a força do grupo de músculos do quadríceps pode ser aplicado. É necessário levantarse cinco vezes, sem ajuda dos braços, em um espaço de tempo cronometrado. Também, pode-se usar o teste cronometrado de suporte de cadeira, contando quantas vezes o paciente consegue levantar-se e sentar-se em trinta segundos. $\underline{\underline{24}}$

b) Quantidade muscular

A quantidade de massa e de músculo pode ser estimada por várias técnicas, requerendo que os resultados sejam ajustados para altura ou IMC. A recomendação é que para aferição da quantidade muscular deve-se empregar a Massa Muscular Esquelética Corporal (MMEC), a Massa Muscular Esquelética Apendicular (MMEA) ou a Área de Secção Transversal de Grupamentos Específicos de Músculos ou Segmentos Corporais. ${ }^{1}$

Entre os métodos diagnósticos para avaliação estão ressonância magnética (RM) e tomografia computadorizada (TC), consideradas padrão-ouro, bem como DEXA, BIA e antropometria. ${ }^{25}$ A RM e TC são os métodos de maior acurácia para quantificação da MMEC, pela vantagem de determinar a qualidade muscular, a massa de gordura e a gordura infiltrada no músculo; porém, têm altos custos, requer pessoal capacitado e não há protocolos bem definidos para a baixa massa muscular. $.2,24$

A DEXA determina a quantidade da massa muscular por meio da massa de tecido magro corporal total e MMEA. ${ }^{25}$ A quantificação da massa muscular, do nível absoluto de MMEC ou MMEA, podem ser ajustados ao tamanho do corpo pelas equações - altura2 (MMEA/altura2); peso (MMEA/peso) ou IMC (MMEA/ IMC). $\underline{26}$ Contudo, não determina a qualidade muscular, devido a capacidade reduzida para diferenciação entre massa magra livre, água e massa óssea, podendo ser influenciada pela hidratação.. ,27 
Avaliação por BIA é um método não-invasivo, prático, reprodutível e relativamente barato, que estima, além dos componentes corporais, a distribuição dos fluidos nos espaços intra-extracelulares, assim como a qualidade, tamanho e integridade celular. Baseiase no princípio da condutividade elétrica para estimativa dos compartimentos corporais (massa gorda e magra). Todavia, não determina a qualidade muscular e a hidratação pode interferir nos resultados. ${ }^{27}$

A antropometria é o método simples e barato, porém apresenta acurácia inferior em relação aos demais, já que não é preciso na aferição da composição e da massa muscular, embora bastante utilizada para avaliar o estado nutricional em idosos. ${ }^{28}$ Robert Lee et al. desenvolveram equações preditivas para a massa muscular, a partir de medidas antropométricas, e identificaram alta correlação com a RM, para estimar a MMEC. $\underline{29}$ Também, Rech et al. identificaram uma alta correlação entre equação preditiva, baseada em medidas antropométricas e a DEXA..$^{30}$ Nesse contexto, a circunferência da panturrilha pode constituir meio de mensuração da massa magra, sendo importante, quando não existem outros métodos acessíveis (ponto de corte $<31 \mathrm{~cm}) . \underline{28}$

A massa muscular pode ser obtida também a partir de valores de creatinina da excreção urinária, visto que é originada quase que exclusivamente do músculo. A dificuldade é manter uma dieta livre de carne por vários dias e uma coleta prolongada da urina, entretanto, a taxa de excreção de creatinina é um promissor protocolo para estimar a MMEC. ${ }^{1}$ A quantidade total ou parcial do potássio dos tecidos moles livres de gordura é outra estratégia para mensurar a massa muscular. Isso porque o músculo esquelético contém mais de $50 \%$ do potássio corporal. É uma mensuração segura e econômica em relação às outras formas, mas é pouco usada na prática. $\frac{31}{}$ c) Desempenho físico

Alguns testes são utilizados para avaliar o desempenho físico, os quais envolvem o aspecto da locomoção, porém, por ser uma aferição multidimensional, envolve não apenas músculos, mas também função nervosa (central e periférica), incluindo o equilíbrio. ${ }^{32} \mathrm{Um}$ destes é o teste de velocidade de marcha de quatro metros, que é um método rápido, seguro e altamente confiável, sendo que valores de velocidade de marcha $\leq 0,8 \mathrm{~m} / \mathrm{s}$ indicam desempenho físico reduzido, podendo indicar sarcopenia grave, caso as variáveis força e massa também estejam reduzidas. ${ }^{1}$

Outros testes utilizados para determinação do desempenho físico são Time Up and Go (TUG), que consiste em sair da posição sentado em uma cadeira, levantar-se, andar três metros, voltar e retornar a postura sentado; o Short Physical Performance Battery (SPPB), composto pelos testes velocidade da marcha, teste de equilíbrio e teste de levantar-se da cadeira; e, o teste de caminhada de 400 metros, que avalia o desempenho físico, averiguando a capacidade de locomoção e a resistência. Esses testes podem demonstrar limitações se o paciente apresentar patologias como demência, distúrbios de marcha ou de equilíbrio, pois inviabilizam sua realização. ${ }^{1.10}$

O EWGSOP2 propôs pontos de cortes respaldados nas populações europeia de adultos jovens e saudáveis $^{33}$, que são apresentados na Tabela $1 .^{1}$

Com a finalidade de rastrear pacientes em risco de sarcopenia, diagnosticá-la e quantificar a gravidade, o algoritmo (Figura 1) torna-se uma ferramenta simples e viável na prática clínica. A árvore decisória chama-se Find-Assess-Confirm-Severity (F-A-C-S) ou Encontrar-Avaliar-Confirmar-Gravidade. 
Tabela 1. Pontos de corte de testes de sarcopenia sugerido pelo EWGSOP2

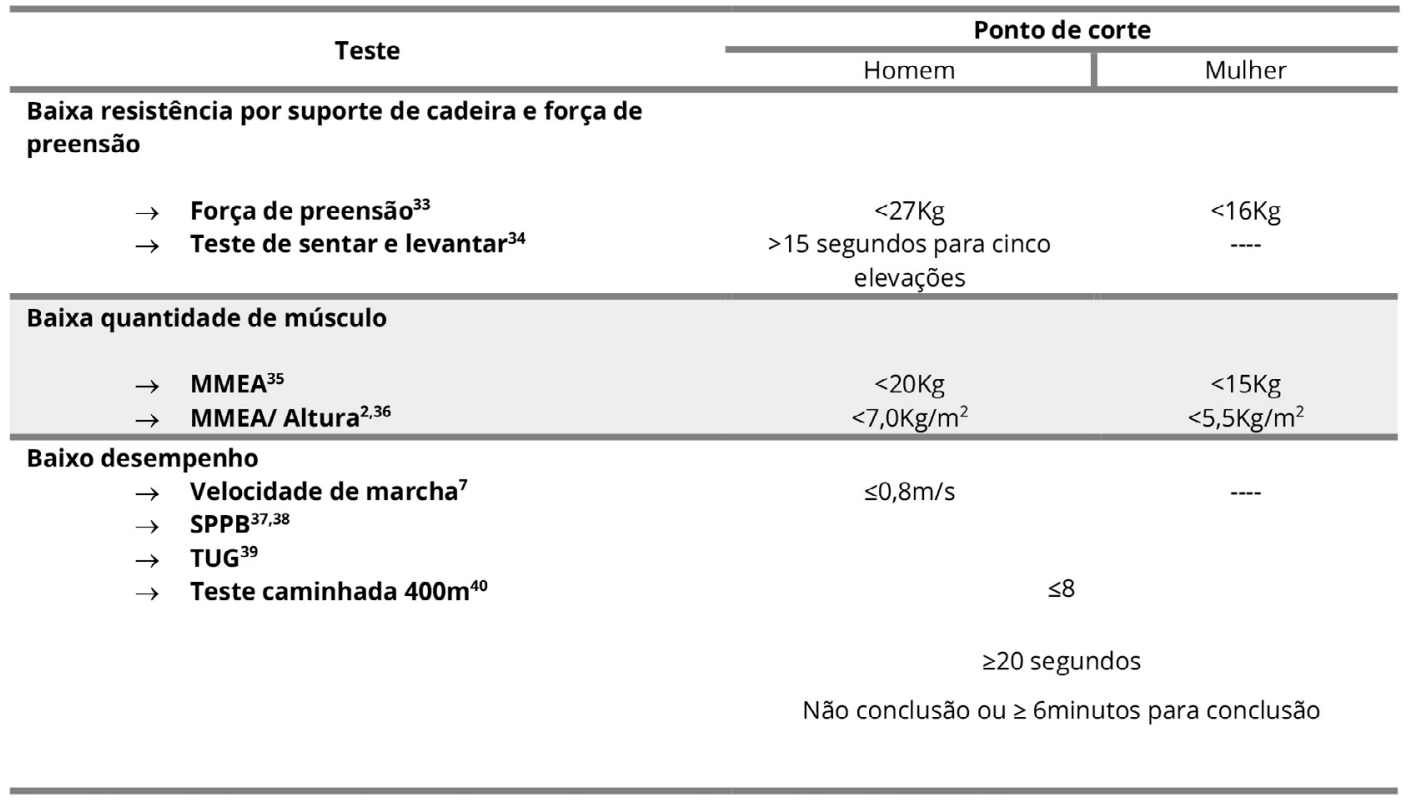

Fonte: Adaptação dos pontos de corte de testes da sarcopenia, sugerido pelo EWGSOP2.

Figura 1. Adaptada do algoritmo sugerido pelo EWGSOP2 ${ }^{1}$

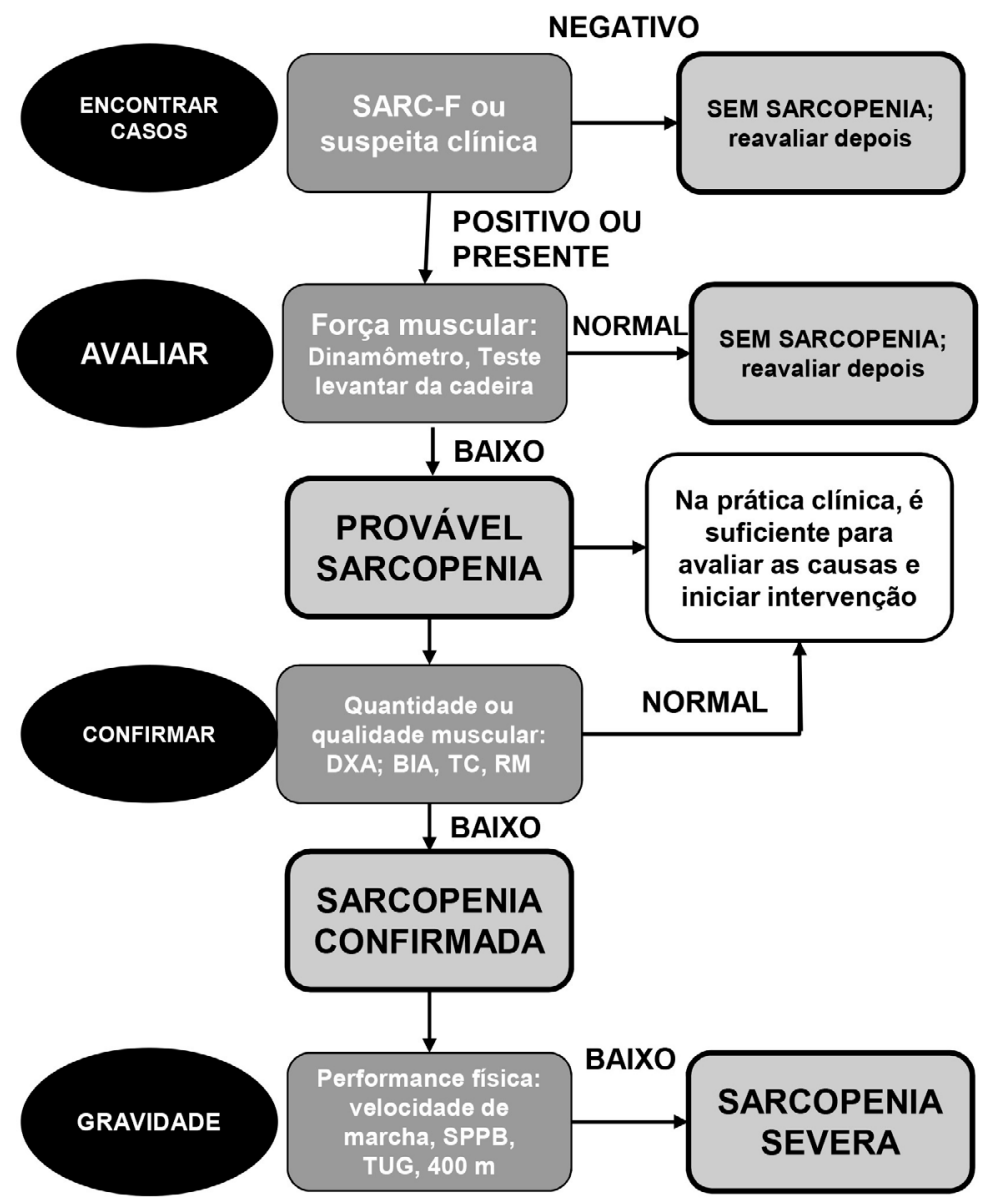




\section{Novos testes para diagnóstico}

Para detecção da sarcopenia, novos testes (Quadro 1) estão em desenvolvimento para aferição de quantidade e qualidade do músculo, assim como da influência desta na qualidade de vida do paciente.

Quadro 1. Novos testes para diagnóstico da sarcopenia

\begin{tabular}{|c|c|}
\hline Nome do teste & Utilização \\
\hline $\begin{array}{l}\text { Imagem da terceira vértebra lombar (L3) por } \\
\text { tomografia computadorizada }\end{array}$ & $\begin{array}{l}\text { Usado para detecção de massa muscular reduzida, já que foi relatado } \\
\text { que o músculo da } L 3 \text { retrata o do corpo. }\end{array}$ \\
\hline Medição do músculo do meio da coxa & $\begin{array}{l}\text { Aplicada em pesquisas, por ser sensível a mudanças e consegue deduzir } \\
\text { a massa muscular esquelética de todo o corpo. É feita através de } \\
\text { ressonância magnética ou tomografia computadorizada. }\end{array}$ \\
\hline $\begin{array}{l}\text { Medição do músculo psoas com tomografia } \\
\text { computadorizada }\end{array}$ & $\begin{array}{l}\text { Apesar de ter sido descrita como um preditor de morbidade em alguns } \\
\text { casos, especialistas debatem que o psoas é um músculo muito pequeno } \\
\text { não representando o estado geral da doença. }\end{array}$ \\
\hline Medição da qualidade muscular & $\begin{array}{l}\text { Avaliada através de tomografia computadorizada e ressonância } \\
\text { magnética, na pesquisa. Com o propósito de verificar mudanças na } \\
\text { composição e funcionamento muscular. }\end{array}$ \\
\hline Teste de diluição de creatina & $\begin{array}{l}\text { Teste utilizado em pesquisas, no qual dose de creatina marcada com } \\
\text { deutério (D3-creatina) é ingerida por via oral pelo paciente em jejum. } \\
\text { Após isso, os tipos de creatina (marcada, não marcada e na urina) são } \\
\text { mensuradas. }\end{array}$ \\
\hline Avaliação ultrassonográfica do músculo & $\begin{array}{l}\text { Incorporado à prática clínica para sustentar diagnóstico de sarcopenia } \\
\text { em adultos mais velhos. O grupo EuGMS sugeriu a avaliação da } \\
\text { espessura muscular, área transversal, comprimento do fascículo, } \\
\text { ângulo de penetração e qualidade. }\end{array}$ \\
\hline $\begin{array}{l}\text { Biomarcadores específicos ou painéis de } \\
\text { biomarcadores }\end{array}$ & $\begin{array}{l}\text { Criação e validação de um biomarcador para diagnóstico e } \\
\text { monitoramento da sarcopenia. }\end{array}$ \\
\hline Questionário SarQoL & $\begin{array}{l}\text { Facilmente aplicável, que pode antever futuras complicações da doença } \\
\text { que afetaram a qualidade de vida do paciente. }\end{array}$ \\
\hline
\end{tabular}

Fonte: Adaptado de EWGSOP2. ${ }^{1}$

\section{Tratamento}

Dentre as formas de tratamento para sarcopenia estão exercício físico, nutrição e reposição hormonal, as quais estão descritas abaixo:

\section{a) Exercício}

A prática de exercícios físicos é uma das formas para amenizar os efeitos catabólicos da inatividade e a consequente sarcopenia. Dentre os diferentes tipos de exercícios, o treino de força promove um grande efeito sobre o aumento da massa e força muscular. Doze semanas de treino de força realizado três vezes por semana resultaram em aumento da força e hipertrofia muscular. ${ }^{10}$ Entretanto, alguns estudos demonstraram melhora na força de maneira similar com uma série por semana. ${ }^{12,41}$

O treinamento de força de forma progressiva é mais utilizado nos idosos. Consiste em realizar exercícios com aumento da resistência até a maior possível, e está associada ao aumento da massa muscular e da função física. ${ }^{42}$ O Colégio Americano de Medicina do Esporte e a Associação Americana do Coração recomendam a realização de 8 a 10 exercícios para os maiores grupos musculares, no mínimo duas vezes não consecutivas por semana, com uma resistência que o indivíduo possa realizar entre 10 e 15 repetições..$^{10}$ 
Apesar de não contribuir para hipertrofia, os exercícios aeróbicos podem aumentar a área transversal das fibras musculares, o volume mitocondrial e a atividade enzimática, promovendo melhora da frequência de declínio na massa muscular e força. Estes reduzem a gordura intramuscular, fortalecem a funcionalidade muscular ${ }^{32}$ e a redução da perda de unidades motoras. ${ }^{43}$ Os efeitos anabólicos do exercício aeróbico estão relacionados ao aumento da oferta de nutrientes para o músculo. $\underline{44}$

Dentre os estudos que demonstraram benefícios da atividade física na sarcopenia em idosos, um revelou que os idosos que a praticam têm uma menor chance de desenvolver sarcopenia e outro evidencia uma melhora do desempenho físico em idosos sarcopênicos submetidos a um programa de exercícios físicos por seis meses. $10,45,46$

\section{b) Nutrição}

Dentre mudanças fisiológicas do envelhecimento estão as alterações da composição corporal, com aumento da massa gorda e gordura visceral e redução involuntária da massa muscular. ${ }^{47}$ Visto que a nutrição se relaciona as causas secundárias da sarcopenia, como: baixa ingestão alimentar, biodisponibilidade reduzida de nutrientes, altas necessidades de nutrientes; portanto, a melhora da dieta e da nutrição podem ser eficazes na prevenção e tratamento da sarcopenia.

Certos nutrientes e padrões alimentares promovem efeitos protetores contra os processos do envelhecimento. $\frac{48}{\mathrm{Um}}$ estímulo anabólico importante para o músculo esquelético é a ingestão de proteínas e aminoácidos. Dentre os aminoácidos, os de cadeia ramificada estão envolvidos na estimulação direta da síntese de proteínas musculares, especificamente a leucina - ativadora de proteínas de sinalização intracelular. E o consumo de forma isolada, suplementada e associada a outros aminoácidos ou carboidratos são eficazes no estímulo da síntese proteica muscular. ${ }^{49}$

No tocante aos micronutrientes, o uso das vitaminas D, C, B6, B12, carotenóide, A e E possuem efeitos sobre o músculo esquelético e se relaciona ao metabolismo e à síntese proteica. Cálcio, selênio, magnésio, potássio, fósforo, ferro e zinco são os minerais utilizados devido à associação destes aos processos de sinalização regulatória para as fibras musculares, proteção muscular contra danos oxidativos, relacionada à atividade física e ao desempenho muscular em idosos, preservação do tecido magro, dentre outras..$^{48}$

Outros fatores dietéticos e nutricionais considerados são as substâncias antioxidantes (ácido graxo ômega-3), que podem ter impacto nos sistemas musculares esqueléticos; combinação de nutrientes que tem relação com a força muscular ou desempenho físico; os grupos de alimentos (laticínios, chás, frutas e vegetais); padrões alimentares; e a avaliação dos fatores dietéticos; todos podem exercer efeitos protetores contra a sarcopenia e fragilidade, bem como desempenhar um papel na preservação da massa muscular e capacidade física. ${ }^{48}$

\section{c) Hormônios}

Considerando a gênese multifatorial da sarcopenia, é razoável acreditar que a suplementação hormonal seja uma boa opção para preveni-la ou tratá-la. Assim sendo, vários hormônios têm efeitos metabólicos sobre massa e função muscular. Dentre eles, estão hormônios sexuais (testosterona, estrogênios - estradiol e dehidroepiandrosterona), cortisol, hormônio do crescimento, IGF-1, grelina, insulina e ocitocina. ${ }^{49}$

\section{Considerações finais}

A sarcopenia é um problema que acomete os indivíduos na senescência, podendo ocorrer precocemente, e é definida como uma doença muscular. Recomendase, para o diagnóstico, a mensuração de força e massa esquelética, além do desempenho físico. Os dados referentes à frequência são bastante divergentes, devido aos diversos instrumentos diagnósticos para mensurar a massa muscular, além dos diferentes pontos de corte. Ademais, demanda custos elevados aos sistemas de saúde, aumenta o risco de hospitalização e as despesas durante a internação.

Para tratá-la, a principal estratégia é o treino de força progressivo, sendo que o exercício aeróbico apresenta efeitos positivos sobre a redução da perda muscular ao longo dos anos, bem como para redução da perda das unidades motoras. A nutrição ideal com uma quantidade adequada de calorias, macro e micronutrientes tem influência sobre a função muscular. 
Como perspectivas futuras, existe a necessidade de verificar as influências que podem causar e piorar a sarcopenia, o momento de intervenção, identificar as pessoas idosas com alto risco de sarcopenia e as ações preventivas. Finalmente, deve ser avaliada a acurácia dos instrumentos de força e desempenho físico para predizer redução de massa muscular esquelética, já que essas talvez sejam as variáveis de maior relevância para o diagnóstico de sarcopenia.

\section{Agradecimentos}

À Universidade do Estado da Bahia (UNEB), ao Conselho Nacional de Desenvolvimento Cientíco e Tecnológico (CNPq)/PIBIC/AF) pela oportunidade e concessão da bolsa para o Programa Institucional de Iniciação Cientíca (IC) a autora Santos NGS, e a Fundação Maria Emília pela concessão de bolsa de apoio à produção cientíca ao autor Camelier AA.

\section{Contribuição dos autores}

Martinez BP, Camelier FWRC, Camelier AA participaram da concepção, delineamento, busca dos dados, interpretação dos resultados, redação do artigo científico, revisão final e submissão para apreciação. Santos NGS, Costa LVM, Santana Neta LG, Sacramento JM e Santos NC participaram da busca de dados, interpretação dos resultados e redação do artigo científico.

\section{Conflitos de interesses}

Nenhum conflito financeiro, legal ou político envolvendo terceiros (governo, empresas e fundações privadas, etc.) foi declarado para nenhum aspecto do trabalho submetido (incluindo, mas não se limitando a subvenções e financiamentos, participação em conselho consultivo, desenho de estudo, preparação de manuscrito, análise estatística, etc.). Camelier AC tem contrato de tempo parcial com a empresa GSK Brasil e recebe bolsa de apoio à Produção Cientíca da Fundação Maria Emília (desde março de 2020).

\section{Referências}

1. Cruz-Jentoft AJ, Bahat G, Bauer J, Boirie $Y$, Bruyère $O$, Cederholm $T$, et al. Sarcopenia: Revised European consensus on definition and diagnosis. Age Ageing. 2019;48(1):16-31. https://doi. org/10.1093/ageing/afy169

2. Morley JE, Abbatecola AM, Argiles JM, Baracos V, Bauer J, Bhasin S, et al. Sarcopenia With Limited Mobility: An International Consensus. J Am Med Dir Assoc. 2011;12(6):403-9. https://doi. org/10.1016/j.jamda.2011.04.014
3. Malmstrom TK, Miller DK, Simonsick EM, Ferrucci L, Morley JE. SARC-F: A symptom score to predict persons with sarcopenia at risk for poor functional outcomes. J Cachexia Sarcopenia Muscle. 2016;7(1):28-36. https://doi.org/10.1002/jcsm.12048

4. Bahat G, Ilhan B. Sarcopenia and the cardiometabolic syndrome: A narrative review. Eur Geriatr Med. 2016;7(3):220-3. https://doi.org/10.1016/j.eurger.2015.12.012

5. Bone AE, Hepgul N, Kon S, Maddocks M. Sarcopenia and frailty in chronic respiratory disease: Lessons from gerontology. Chron Respir Dis. 2017;14(1):85-99. https://doi. org/10.1177/1479972316679664

6. Chang KV, Hsu TH, Wu WT, Huang KC, Han DS. Association Between Sarcopenia and Cognitive Impairment: A Systematic Review and Meta-Analysis. J Am Med Dir Assoc. 2016;17(12):1164. e7-1164.e15. https://doi.org/10.1016/j.jamda.2016.09.013

7. Beaudart $C$, Reginster JY, Geerinck $A$, Locquet $M$, Bruyère O. Current review of the SarQoL®: a health-related quality of life questionnaire specific to sarcopenia. Expert Rev Pharmacoeconomics Outcomes Res. 2017;17(4):335-41. https:// doi.org/10.1080/14737167.2017.1360768

8. Steffl M, Bohannon RW, Sontakova L, Tufano JJ, Shiells K, Holmerova I. Relationship-between-sarcopenia-and-physicalactivity-in-the. Clin Interv Aging. 2017;12:835-45. https://doi. org/10.2147/CIA.S132940

9. Mitchell WK, Williams J, Atherton P, Larvin M, Lund J, Narici $M$, et al. Sarcopenia, dynapenia, and the impact of advancing age on human skeletal muscle size and strength; a quantitative review. Front Physiol. 2012;3:260. https://doi.org/10.3389/ fphys.2012.00260

10. Martinez BP, Camelier FWR, Camelier AA. Sarcopenia em Idosos: Um estudo de revisão. Rev Pesq Fisioter. 2014;2(1):62-70. https://doi.org/10.17267/2238-2704rpf.v4i1.349

11. Rom O, Kaisari S, Aizenbud D, Reznick AZ. Lifestyle and Sarcopenia - Etiology, Prevention and Treatment. Rambam Maimonides Med J. 2012;3(4):e0024. https://doi.org/10.5041/ RMMJ.10091

12. Pedersen BK. The diseasome of physical inactivity - and the role of myokines in muscle-fat cross talk. J Physiol. 2009;587(23):5559-68. https://doi.org/10.1113/ jphysiol.2009.179515

13. Newman AB, Kupelian V, Visser M, Simonsick EM, Goodpaster $\mathrm{BH}$, Kritchevsky SB, et al. Strength, but not muscle mass, is associated with mortality in the health, aging and body composition study cohort. Gerontologist. 2006;61(1):72-7. https:// doi.org/10.1093/gerona/61.1.72 
14. Shimokata H, Shimada H, Satake S, Endo N, Shibasaki K, Ogawa S, et al. Chapter 2 Epidemiology of sarcopenia. Geriatr Gerontol Int. 2018;18(S1):13-22. https://doi.org/10.1111/ggi.13320

15. Morley JE, Anker SD, von Haehling S. Prevalence, incidence, and clinical impact of sarcopenia: facts, numbers, and epidemiology-update 2014. J Cachexia Sarcopenia Muscle. 2014;5(4):253-9. https://doi.org/10.1007/s13539-014-0161-y

16. Diz JBM, Queiroz BZ, Tavares LB, Pereira LSB. Prevalência de sarcopenia em idosos: resultados de estudos transversais amplos em diferentes países. Rev Bras Geriatr Gerontol. 2015;18(3):66578. https://doi.org/10.1590/1809-9823.2015.14139

17. Alexandre TS, Duarte YAO, Santos JLF, Lebrão ML. Prevalência e fatores associados à sarcopenia, dinapenia e sarcodinapenia em idosos residentes no Município de São Paulo - Estudo SABE. Rev Bras Epidemiol. 2018;21(Suppl 2):e180009. https://doi. org/10.1590/1980-549720180009.supl.2

18. Confortin SC, Ono LM, Barbosa AR, D'orsi E. Sarcopenia e sua associação com mudanças nos fatores socioeconômicos, comportamentais e de saúde: Estudo EpiFloripa Idoso. Cad Saude Publica. 2018;34(12):e00164917. https://doi.org/10.1590/0102$\underline{311 \times 00164917}$

19. Moreira VG, Perez M, Lourenço RA. Prevalence of sarcopenia and its associated factors: The impact of muscle mass, gait speed, and handgrip strength reference values on reported frequencies. Clinics. 2019;74(7): e477. https://doi.org/10.6061/clinics/2019/ $\underline{\mathrm{e} 477}$

20. Zhang X, Xie X, Dou Q, Liu C, Zhang W, Yang Y, et al. Association of sarcopenic obesity with the risk of all-cause mortality among adults over a broad range of different settings: A updated meta-analysis. BMC Geriatr. 2019;19(1):183. https://doi. org/10.1186/s12877-019-1195-y

21. Reis MM, Arantes PMM. Medida da força de preensão manual- validade e confiabilidade do dinamômetro saehan. Fisioter Pesq. 2011;18(2):176-81. https://doi.org/10.1590/S180929502011000200013

22. Francis $\mathrm{P}$, Toomey C, Mc Cormack W, Lyons M, Jakeman P. Measurement of maximal isometric torque and muscle quality of the knee extensors and flexors in healthy 50- to 70-year-old women. Clin Physiol Funct Imaging. 2017;37(4):448-55. https://doi. org/10.1111/cpf.12332

23. Masanés F, Luque XR, Salvà A, Serra-Rexach JA, Artaza I, Formiga $F$, et al. Cut-off points for muscle mass - not grip strength or gait speed - determine variations in sarcopenia prevalence. J Nutr Health Aging. 2017;21(7):825-9. https://doi. org/10.1007/s12603-016-0844-5

24. Buckinx F, Landi F, Cesari M, Fielding RA, Visser M, Engelke $K$, et al. Pitfalls in the measurement of muscle mass: a need for a reference standard. J Cachexia Sarcopenia Muscle. 2018;9(2):26978. https://doi.org/10.1002/jcsm.12268
25. Kim KM, Jang HC, Lim S. Differences among skeletal muscle mass indices derived from height-, weight-, and body mass indexadjusted models in assessing sarcopenia. Korean J Intern Med. 2016;31(4):643-50. https://doi.org/10.3904/kjim.2016.015

26. Thomas DR. Sarcopenia. Clin Geriatr Med. 2010;26(2):331-46. https://doi.org/10.1016/j.cger.2010.02.012

27. Tosato M, Marzetti E, Cesari M, Savera G, Miller RR, Bernabei R, et al. Measurement of muscle mass in sarcopenia: from imaging to biochemical markers. Aging Clin Exp Res. 2017;29(1):19-27. https://doi.org/10.1007/s40520-016-0717-0

28. Lee RC, Wang Z, Heo M, Ross R, Janssen I, Heymsfield SB. Total-body skeletal muscle mass: development and crossvalidation of anthropometric prediction models. Am. J. Clin. Nutr. 2000;72(3):796-803. https://doi.org/10.1093/ajcn/72.3.796

29. Rech CR, Dellagrana RA, Marucci MFN, Petroski EL. Validade de equações antropométricas para estimar a massa muscular em idosos. Rev Bras Cineantropom Desempenho Hum. 2012;14(1):23-31. https://doi.org/10.5007/1980$\underline{0037.2012 v 14 n 1 p 23}$

30. Wielopolski L, Ramirez LM, Gallagher D, Heymsfield SB, Wang ZM. Measuring partial body potassium in the arm versus total body potassium. J Appl Physiol. 2006;101(3):945-9. https://doi. org/10.1152/japplphysiol.00999.2005

31. Beaudart C, Rolland Y, Cruz-Jentoft AJ, Bauer JM, Sieber C, Cooper C, et al. Assessment of Muscle Function and Physical Performance in Daily Clinical Practice: A position paper endorsed by the European Society for Clinical and Economic Aspects of Osteoporosis, Osteoarthritis and Musculoskeletal Diseases (ESCEO). Calcif Tissue Int. 2019;105(1):1-14. https://doi. org/10.1007/s00223-019-00545-w

32. Dodds RM, Syddall HE, Cooper R, Benzeval M, Deary IJ, Dennison EM, et al. Grip strength across the life course: Normative data from twelve British studies. PLoS One. 2014;9(12):e113637. https://doi.org/10.1371/journal. pone. 0113637

33. Cesari M, Kritchevsky SB, Newman AB, Simonsick EM, Harris TB, Penninx BW, et al. Added value of physical performance measures in predicting adverse health-related events: results from the Health, Aging and Body Composition Study. J Am Geriatr Soc. 2009;57(2):251-9. https://doi.org/10.1111/j.15325415.2008.02126.x

34. Studenski SA, Peters KW, Alley DE, Cawthon PM, McLean RR, Harris TB, et al. The FNIH Sarcopenia Project: Rationale, Study Description, Conference Recommendations, and Final Estimates. Gerontologist. 2014;69(5):547-58. https://doi.org/10.1093/gerona/ glu010 
35. Gould H, Brennan SL, Kotowicz MA, Nicholson GC, Pasco JA. Total and Appendicular Lean Mass Reference Ranges for Australian Men and Women: The Geelong Osteoporosis Study. Calcif. 2014;94(4):363-72. https://doi.org/10.1007/s00223-0139830-7

36. Distefano G, Standley RA, Zhang X, Carnero EA, Yi F, Cornnell $\mathrm{HH}$, et al. Physical activity unveils the relationship between mitochondrial energetics, muscle quality, and physical function in older adults. J Cachexia Sarcopenia Muscle. 2018;9(2):279-94. https://doi.org/10.1002/jcsm.12272

37. Ruan XY, Gallagher D, Harris T, Albu J, Heymsfield S, Kuznia $P$, et al. Estimating whole body intermuscular adipose tissue from single cross-sectional magnetic resonance images. J Appl Physiol. 2007;102(2):748-54. https://doi.org/10.1152/ japplphysiol.00304.2006

38. Woo J, Leung J, Morley JE. Defining Sarcopenia in Terms of Incident Adverse Outcomes. J Am Med Dir Assoc. 2015;16(3):24752. https://doi.org/10.1016/j.jamda.2014.11.013

39. Bahat G, Yilmaz O, Kiliç C, Oren MM, Karan MA. Performance of SARC-F in Regard to Sarcopenia Definitions, Muscle Mass and Functional Measures. J Nutr Health Aging. 2018;22(8):898-903. https://doi.org/10.1007/s12603-018-1067-8

40. Taaffe DR, Duret C, Wheeler S, Marcus R. Once-weekly resistance exercise improves muscle strength and neuromuscular performance in older adults. J Am Geriatr Soc. 1999;47(10):120814. https://doi.org/10.1111/j.1532-5415.1999.tb05201.x

41. Mangione KK, Miller AH, Naughton IV. Cochrane review: Improving physical function and performance with progressive resistance strength training in older adults. Phys Ther. 2010;90(12):1711-5. https://doi.org/10.2522/ptj.20100270
42. Power GA, Dalton BH, Behm DG, Doherty TJ, Vandervoort AA, Rice CL. Motor unit survival in lifelong runners is muscle dependent. Med Sci Sports Exerc. 2012;44(7):1235-42. https://doi. org/10.1249/MSS.0b013e318249953c

43. Timmerman KL, Dhanani S, Glynn EL, Fry CS, Drummond $M J$, Jennings $K$, et al. A moderate acute increase in physical activity enhances nutritive flow and the muscle protein anabolic response to mixed nutrient intake in older adults. Am J Clin Nutr. 2012;95(6):1403-12. https://doi.org/10.3945/ajcn.111.020800

44. Akune T, Muraki S, Oka H, Tanaka S, Kawaguchi H, Nakamura $\mathrm{K}$, et al. Exercise habits during middle age are associated with lower prevalence of sarcopenia: The ROAD study. Osteoporos Int. 2014;25(3):1081-8. https://doi.org/10.1007/s00198-013-2550-z

45. Liu CK, Leng X, Hsu FC, Kritchevsky SB, Ding J, Earnest CP, et al. The impact of sarcopenia on a physical activity intervention: The lifestyle interventions and independence for elders pilot study (LIFE-P). J Nutr Health Aging [Internet]. 2014;18(1):59-64. Disponível em: https://www.ncbi.nlm.nih.gov/pmc/articles/ PMC4111145/

46. Margutti KMM, Schuch NJ, Schwanke CHA. Marcadores inflamatórios, sarcopenia e seus critérios diagnósticos em idosos: uma revisão sistemática. Rev bras geriatr gerontol. 2017;20(3):441-53. https://doi.org/10.1590/1981$\underline{22562017020.160155}$

47. Ganapathy A, Nieves JW. Nutrition and sarcopenia-What do we know? Nutrients. 2020;12(6):1755. https://doi.org/10.3390/ nu12061755

48. Makanae $Y$, Fujita S. Role of exercise and nutrition in the prevention of sarcopenia. J Nutr Sci Vitaminol. 2015;61:S125-7. https://doi.org/10.3177/jnsv.61.5125

49. Morley JE, Malmstrom TK. Frailty, Sarcopenia, and Hormones. Endocrinol Metab Clin North Am. 2013;42(2):391-405. http:// dx.doi.org/10.1016/j.ecl.2013.02.006 